\title{
Intrinsic Spin Hall Edges
}

\author{
İnanç Adagideli* and Gerrit E. W. Bauer \\ Kavli Institute of Nanoscience, TU Delft, Lorentzweg 1, 2628 CJ Delft, The Netherlands
}

(Received 30 June 2005; published 15 December 2005)

\begin{abstract}
The prediction of intrinsic spin Hall currents by Murakami et al. and Sinova et al. raised many questions about methods of detection and the effect of disorder. We focus on a contact between a Rashbatype spin-orbit coupled region with a normal two-dimensional electron gas and show that the spin Hall currents, though vanishing in the bulk of the sample, can be recovered from the edges. We also show that the current-induced spin accumulation in the spin-orbit coupled system diffuses into the normal region and contributes to the spin current in the leads.
\end{abstract}

DOI: 10.1103/PhysRevLett.95.256602

PACS numbers: 72.25.Dc, 72.20.Dp, 72.25.Mk

Transport and manipulation of spins in semiconductor structures have become a mainstream in condensed matter physics [1]. In principle, spins can be injected into semiconductors by ferromagnets via electric contacts. However, finding suitable material combinations that do not suffer from the conductance mismatch [2] turned out to be difficult. Furthermore, introducing ferromagnetic materials into the semiconductor microfabrication process is undesirable from a technological point of view. The prospect to generate spin accumulations in semiconductors without ferromagnets or applied magnetic fields simply by driving a current through a material with intrinsic spin-orbit (SO) interaction and broken inversion symmetry [3-5] is therefore very attractive. A related effect that attracted a lot of attention is the spin Hall effect (SHE), i.e., the spin current (SC) that has been predicted to flow normal to an applied electric current in the absence of an applied magnetic field. When caused by impurities with spin-orbit scattering [6,7] this effect is called "extrinsic." A spin Hall current (SHC) can also be generated by the spin-orbit interaction of the lattice potential as has recently been predicted for $p$-doped III-V semiconductors [8] and the two-dimensional electron gas with a Rashba-type SO interaction (R2DEG) [9]. Whether the experimental observations of the spin Hall effect by optical methods [10] have an intrinsic or extrinsic origin is still a matter of debate. In spite of initial controversies, analytic theories [11-16] as well as numerical simulations [17,18] consistently predict that the SHE should vanish in the disordered (bulk) R2DEG [19]. Some doubts remain whether the SC, being a nonconserved quantity in SO coupled systems, is observable at all [20].

In this Letter, we focus on the spin currents near normal contacts. First, an elementary and general proof is given that the spin Hall effect due to the lattice SO coupling (viz. intrinsic SHE) must vanish in diffuse bulk systems with an arbitrarily strong SO interaction that is linear in the electron wave vector. Nevertheless, using an extension of this argument to finite system sizes, we show that near the edges a spin Hall current can persist. Next, by solving the kinetic equations for a model system of a R2DEG in contact with a normal metal system without SO interaction, we calculate indeed a finite SHC. This SC is generated in a skin depth determined by the Dyakonov-Perel [6] spin-flip diffusion length $\left(L_{s}\right)$ and the polarization is not normal to the 2DEG, having a component due to the diffusion current from the SO-generated spin accumulation (SA). The magnitude of the SC generated at the edges depends on whether the system is clean (impurity broadening less than the SO splitting) or dirty (opposite limit). However, in contrast to the bulk SC, the edge SC does not vanish when the system is not ballistic ( $L_{s}$ smaller than the system size). The SC is calculated in the normal metal contact and is therefore certainly a transport current [20]. Related work on interface and boundary effects focused so far on mesoscopic systems via numerical simulations [21] and the SA near hard wall boundaries [22].

We proceed to derive a transport equation valid in the Boltzmann limit that is capable of handling the full spin dynamics. In $2 \times 2$ spin space, the Hamiltonian is

$$
H=\mathbf{p}^{2} / 2 m+V(\mathbf{x})+H^{R}-e \boldsymbol{E}(t) \cdot \mathbf{x},
$$

where $\mathbf{x}$ and $\mathbf{p}$ are the (two-dimensional) position and momentum operators, respectively. Here the unit vector $\hat{z}$ is normal to the 2DEG, $H^{R}=(\alpha / \hbar) \mathbf{p} \cdot(\boldsymbol{\sigma} \times \hat{z})$ is the Rashba Hamiltonian with Pauli matrices $\boldsymbol{\sigma}$ and $\alpha$ parametrizes the strength of the SO interaction [23], $\boldsymbol{E}$ is the electric field, and $V(\mathbf{x})=\sum_{i=1}^{N} \phi\left(\mathbf{x}-\boldsymbol{X}_{i}\right)$ is the impurity potential, modeled by $N$ impurity centers located at points $\left\{\boldsymbol{X}_{i}\right\}$. Although it is possible to consider ac fields, we focus here on dc fields in the $x$ direction and assume that the electric field is turned on adiabatically in the remote past at which the system was in thermal equilibrium; i.e., we assume $\boldsymbol{E}=\lim _{s \rightarrow 0} \boldsymbol{E}_{0} \exp (s t / \hbar)$. To leading order in the impurity potential the diagonal elements of the density matrix in reciprocal space satisfy the following equation [24]:

$$
\begin{aligned}
-i s f(\boldsymbol{k})+\left[f(\boldsymbol{k}), H_{k}^{R}\right]= & \sum_{k^{\prime}}\left(f_{\boldsymbol{k} \boldsymbol{k}^{\prime}} V_{\boldsymbol{k}^{\prime} \boldsymbol{k}}-V_{\boldsymbol{k} \boldsymbol{k}^{\prime}} f_{\boldsymbol{k}^{\prime} \boldsymbol{k}}\right) \\
& +e \boldsymbol{E} \cdot\left[\boldsymbol{x}, f^{0}\right] .
\end{aligned}
$$

Here $f^{0}=\left(f_{+}^{0}+f_{-}^{0}\right) / 2+\sigma_{\theta}\left(f_{+}^{0}-f_{-}^{0}\right) / 2$ is the equilib- 
rium density matrix with $f_{ \pm}^{0}(k)=\mathcal{F}\left(\hbar^{2} k^{2} / 2 m \pm \alpha k\right)$, where $\mathcal{F}(E)$ is the Fermi function and $\sigma_{\theta}=\mathbf{k} \cdot(\boldsymbol{\sigma} \times$ $z) / k$. The off-diagonal elements of the density matrix read

$$
f_{\boldsymbol{k} \boldsymbol{k}^{\prime}}=\frac{i}{2 \pi} \int_{-\infty}^{\infty} d E G_{\boldsymbol{k}}^{R}(E)\left(f(\boldsymbol{k})-f\left(\boldsymbol{k}^{\prime}\right)\right) G_{\boldsymbol{k}^{\prime}}^{A}(E) V_{\boldsymbol{k} \boldsymbol{k}^{\prime}}
$$

Here $G_{k}^{R(A)}(E)=\left[E-H_{0}-H_{k}^{R}+(-) i s / 2\right]^{-1}$ are retarded (advanced) matrix Green functions. Substituting Eq. (3) into Eq. (2) and averaging over $V_{k k^{\prime}}$ (in the Boltzmann limit averaging is equivalent to replacing $\left|V_{k k^{\prime}}\right|^{2}$ with its average value $N\left|\phi_{k k^{\prime}}\right|^{2} / A^{2}$, where $N$ is the number of impurities and $A$ is the area [24]) gives our basic equation, valid for weak $V$ and low enough impurity densities to ignore weak localization effects, but to all orders in $\alpha$.

The mechanism behind the intrinsic SHE is the spin precession of quasiparticles while being accelerated by the electric field [9]. However, impurity scattering provides a brake that in the steady state cancels the acceleration on average. Therefore the SHE should vanish in an infinite, homogeneously disordered system. This idea can be formally expressed by considering the acceleration operator $\ddot{x}_{i}=e E_{i} / m-\nabla_{i} V / m-2 \alpha^{2} \hbar^{-3} \epsilon_{3 j i} \sigma_{3} p_{j}$, where $\epsilon_{i j k}$ is the antisymmetric tensor and the Einstein summation convention is implied. We notice that the last term is proportional to the $j^{\prime}$ th component of the SC operator polarized in the $z$ direction, $J_{j}^{z}=\left\{v_{j}, \sigma_{z}\right\}$, where $\{$,$\} is the anticommu-$ tator. The expectation value is defined by $\langle O\rangle \equiv \overline{\operatorname{Tr} f O}$, where the trace is over wave vector and spin space, and $\cdots$ denotes averaging with respect to impurity configurations. In a steady state the average acceleration $\left\langle\ddot{\mathbf{x}}_{i}\right\rangle$ must vanish, leading to the equality

$$
2 \alpha^{2} m^{2} \hbar^{-3} \epsilon_{3 j i}\left\langle J_{j}^{z}\right\rangle=e E_{i}-\left\langle\nabla_{i} V\right\rangle .
$$

We show that the right-hand side of this equality also vanishes by evaluating the expectation value of the deceleration due to impurity scattering:

$$
\begin{aligned}
\left\langle\nabla_{i} V\right\rangle & =-i \sum_{k k^{\prime}}\left(k_{i}-k_{i}^{\prime}\right) V_{k k^{\prime}} \operatorname{tr} f_{\boldsymbol{k}^{\prime} \boldsymbol{k}} \\
& =-i \sum_{k} \operatorname{tr}\left(k_{i}\left[f(\boldsymbol{k}), H_{\boldsymbol{k}}^{R}\right]+i k_{i} e \boldsymbol{E}_{j} \nabla_{k_{j}} f^{0}\right)=e \boldsymbol{E}_{i},
\end{aligned}
$$

where tr is the trace over spin components and Eqs. (2) and (3) have been used in the second step. Substituting the expression above into Eq. (4) we see that all components of the SC polarized in the $z$ direction vanish with the average acceleration [25]. This result holds for infinite systems regardless of the range of the impurity potential or whether the system is clean $\left(\alpha k_{F} \tau / \hbar \gg 1\right)$ or dirty $\left(\alpha k_{F} \tau / \hbar \ll 1\right)$, where $\tau$ is the momentum lifetime. This generalizes previous results [11-16]. However, as we show below, for semi-infinite and finite systems, SCs persist near the edges, but the size of these currents depends on whether the system is clean or dirty.
This line of argument allows one to check related Hamiltonians. In the presence of $k$-linear Dresselhaus and Rashba terms, the result remains unchanged besides the substitution $\alpha^{2} \rightarrow \alpha^{2}-\beta^{2}$, where $\beta$ is the Dresselhaus spin-orbit coupling constant. Thus the SHC still vanishes (with the possible exception of the degeneracy point $\alpha=\beta$ [26]). When the SO coupling contains cubic terms like $\alpha(k)=\alpha_{0}+\alpha_{1} k^{2}$, it is easy to show that the SHC is proportional to $\alpha_{1}$ [12]. Another possible situation is the presence of a Zeeman field: in this case the operator equation is modified to give $\alpha \epsilon_{3 j i}\left\langle J_{j}^{z}\right\rangle=$ $\left\langle\sigma_{i}\right\rangle B_{3}-\left\langle\sigma_{3}\right\rangle B_{i}$, relating the SHC to the SA. If $\alpha$ varies in space, the SHC is found to be proportional to the spatial derivatives of $\alpha$ and $f$. If $\alpha$ is constant, but $f$ varies, e.g., due to boundaries or interfaces [13], the SHC is proportional to the gradients of the density matrix:

$$
\frac{4 \alpha^{2} m^{2}}{\hbar^{3}} \epsilon_{3 j i}\left\langle J_{j}^{z}\right\rangle=\left\langle k_{i}\left\{\frac{\hbar^{2} k_{l}}{m}+\alpha(\hat{z} \times \boldsymbol{\sigma})_{l}, \nabla_{l} f(\boldsymbol{k}, \boldsymbol{x})\right\}\right\rangle .
$$

This equation shows that although the bulk SH current vanishes, there is no a priori reason for SH currents near the edges of the R2DEG to vanish. Next, we show indeed the SH currents do not vanish near the edges. We therefore return to the quantum transport equation, allowing for spatially varying density matrices but assuming shortrange $s$-wave scatterers with $\overline{\left|V_{k k^{\prime}}\right|^{2}}=N \lambda^{2} / A$ and $\alpha / k_{F} \ll 1$. We solve the transport equation by expressing $f$ in terms of a gradient expansion of $\rho(E)=$ $\left(i \hbar^{2} / 2 \pi m\right) \sum_{k}\left(G_{k}^{R}(E) f(\boldsymbol{k})-f(\boldsymbol{k}) G_{k}^{A}(E)\right)$. In the case of $s$-wave scatterers and to leading order in $m \alpha / \hbar^{2} k_{F}$, this generates the same diffusion equation as Ref. [13] and Burkov et al. in Ref. [9]. In terms of components of the density matrix, $\rho=n+\mathbf{s} \cdot \boldsymbol{\sigma}+\sigma_{3} s_{3}$ :

$$
\begin{gathered}
D \nabla^{2} n-4 K_{s-c}(\boldsymbol{\nabla} \times \mathbf{s})_{z}=0, \\
D \nabla^{2} s_{3}-2 K_{p}(\boldsymbol{\nabla} \cdot \mathbf{s})=\frac{2 s_{3}}{\tau_{s}}, \\
D \nabla^{2} \mathbf{s}+2 K_{p} \boldsymbol{\nabla} s_{3}-K_{s-c}(\mathbf{z} \times \boldsymbol{\nabla}) n=\frac{\mathbf{s}}{\tau_{s}} .
\end{gathered}
$$

Here $\quad D=v_{F}^{2} \tau / 2, \quad \tau_{s}=\tau\left(1+4 \xi^{2}\right) / 2 \xi^{2}, \quad K_{s-c}=$ $\alpha \xi^{2} /\left(1+4 \xi^{2}\right), \quad K_{p}=\hbar k_{F} \xi / m\left(1+4 \xi^{2}\right)^{2} \quad$ and $\xi=$ $\alpha k_{F} \tau / \hbar$. The $\mathrm{SC}$ is given, in the diffuse limit by

$$
\begin{aligned}
j_{j}^{i}= & \frac{v_{F} \xi}{1+4 \xi^{2}}\left[\delta_{i 3}\left(s_{j}-\epsilon_{j m 3} \frac{\alpha \tau}{2} \nabla_{m} n\right)-\delta_{i j} s_{3}\right] \\
& -D \nabla_{j} s_{i} .
\end{aligned}
$$

Electric field dependence can be reintroduced by the substitution $\boldsymbol{\nabla} \rightarrow \boldsymbol{\nabla}+e \mathbf{E} \partial_{E}$.

We now focus on a four terminal structure as depicted in Fig. 1. This structure consists of two massive reservoirs biased to produce a charge current in the $x$ direction. Between the reservoirs there is a R2DEG hybrid structure, 


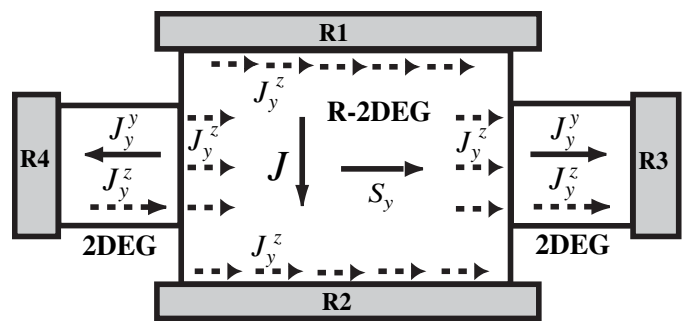

FIG. 1. The schematic setup for SC generation in an R2DEG that is current biased by reservoirs R1 and R2 and equipped with 2DEG Hall contacts with vanishing spin-orbit coupling to reservoirs R3 and R4. The latter can be magnetic or nonmagnetic and voltage biased such that no charge current flows through the 2DEGs. The dashed arrows indicate the local SHC densities that are concentrated near the interfaces. The dominant part of the spin current flowing into R3 (or R4) is generated within a skin depth $L_{s}$ near the corresponding interface and contains a diffusion term from the SA $S_{y}$ in the bulk of the R2DEG. The contribution of the SHC density near R1 and R2 to the net SC [13] flowing into R3 (or R4) is exponentially small.

with $\alpha(\mathbf{x})=\alpha_{0}$ for $L>y>0$ and $\alpha(\mathbf{x})=0$ for $y<0$ and $y>L$, and additional differences are disregarded. The normal 2DEGs are coupled to massive reservoirs R3 and $\mathrm{R} 4$, which are biased such that the charge current is zero, but a SC can still be collected. To the order (in $\alpha / k_{F}$ ) that we are considering, $n$ does not depend on $y$. Alternatively, one can assume that the transverse size of the leads to R3 and $\mathrm{R} 4$ are much smaller than the distance between R1 and $\mathrm{R} 2$, in which case one can also neglect the $y$ dependence of $n$. We consider the distributions at a safe distance from the reservoirs R1 and R2. In this region $s_{y}$ and $s_{z}$ depend only on $y$ and $s_{x}=0$ and the diffusion equation becomes

$$
\left(\begin{array}{cc}
\frac{d^{2}}{d \bar{y}^{2}}-1 & 2 \eta \frac{d}{d \bar{y}} \\
-2 \eta \frac{d}{d \bar{y}} & \frac{d^{2}}{d \bar{y}^{2}}-2
\end{array}\right)\left(\begin{array}{c}
s_{2} \\
s_{3}
\end{array}\right)=\left(\begin{array}{c}
\tau_{s} K_{s-c} \frac{d n}{d x} \\
0
\end{array}\right),
$$

where $\eta=\left(1+4 \xi^{2}\right)^{-3 / 2}, \bar{y}=y / L_{s}$, and $L_{s}=\sqrt{D} \bar{\tau}_{s}$.

In order to derive the matching condition for the spin and charge distribution functions at the contacts (i.e., interface between the R2DEG and 2DEG), short-range fluctuations of boundaries and interfaces that can lead to additional spin relaxation [27] are disregarded. We consider an arbitrary solution $\chi$ of the Schrödinger equation set by the Hamiltonian Eq. (1). We label the solutions in the R2DEG and 2DEG regions $\chi_{R}$ and $\chi_{N}$, respectively. At the interface $\left.\chi_{R}\right|_{0}=\left.\chi_{N}\right|_{0}$ and $\left.\mathbf{n} \cdot\left[i \boldsymbol{\nabla}+\alpha_{0}(\mathbf{z} \times \sigma)\right] \chi_{R}\right|_{0}=$ $\left.i \mathbf{n} \cdot \boldsymbol{\nabla} \chi_{N}\right|_{0}$, where $\mathbf{n}$ is the unit vector normal to the interface. Multiplying from the left with $\left.\chi\right|_{0} ^{\dagger} \sigma_{i}$ and evaluating the imaginary part we obtain

$$
\begin{aligned}
&\left.\mathbf{n} \cdot\left[i \chi_{N}^{\dagger} \sigma_{i} \boldsymbol{\nabla} \chi_{N}-i\left(\nabla_{\chi_{N}}\right)^{\dagger} \sigma_{i} \chi_{N}\right]\right|_{0} \\
&=\mathbf{n} \cdot\left[i \chi_{R}^{\dagger} \sigma_{i} \nabla_{\chi_{R}}-i\left(\nabla_{\chi_{R}}\right)^{\dagger} \sigma_{i} \chi_{R}\right. \\
&\left.+\alpha_{0} \chi_{R}^{\dagger}\left\{\sigma_{i},(\mathbf{z} \times \boldsymbol{\sigma})\right\} \chi_{R}\right]\left.\right|_{0} .
\end{aligned}
$$

We identify the right- (left-) hand side of this equation as the SC density in the Rashba (normal) 2DEG. In terms of spin density matrices we have $\operatorname{tr}\left(f^{R}\left\{\sigma_{i}, \mathbf{n} \cdot \mathbf{j}(0)\right\}\right)=$ $\operatorname{tr}\left(f^{N}\left\{\sigma_{i}, \mathbf{n} \cdot \mathbf{j}(0)\right\}\right)$, where $\mathbf{j}(\mathbf{x}) \equiv\{\mathbf{v}, \delta(\hat{\mathbf{x}}-\mathbf{x})\}$ is the local current density operator. We therefore have to match the normal components of the SC density given by Eq. (9) at the interface [28]. Since the operator $\left\{\mathbf{v}_{i}, \boldsymbol{\sigma}\right\} j$ can have a nonzero expectation value in the equilibrium state it has been questioned whether it governs transport of spins in the presence of SO interaction [20]. We notice that the negative energy solutions (relative to the band crossing) of the Hamiltonian Eq. (1) without the electric field term are localized to the R2DEG region if surrounded by a region with $\alpha=0$. In a normal 2DEG surrounding the R2DEG, we can therefore show that equilibrium SCs exposed in Ref. [20] do not transmit into the normal region. Moreover, the expectation value of the SC density operator vanishes for these localized solutions, and it is precisely the absence of contributions from these solutions that shifts the equilibrium value of the SC to zero.

Returning to the setup in Fig. 1, we assume that the reservoirs R3 and R4 are sufficiently large such that all components of the SA at their respective interfaces with the ordinary 2DEG leads vanish. Shrinking the widths of the 2DEGs to zero, we obtain the effective boundary conditions $s_{i}=0$ at the R2DEG|R3 interface. The finite Ohmic resistance of a finite 2DEG region between the R2DEG and the reservoir can easily be reintroduced if necessary and would lead to somewhat smaller spin conductances. We then can solve the diffusion equation above and obtain the spin current using Eq. (9).

Analytical formulas turn out to be too lengthy to reproduce here. Our results are therefore summarized in Fig. 2. The SA is suppressed at the interface, reflecting the massive-reservoir boundary condition. The gradient of the two components $s_{y}$ and $s_{z}$ leads to two SC components. The SC polarized in the $y$ direction represents the out-

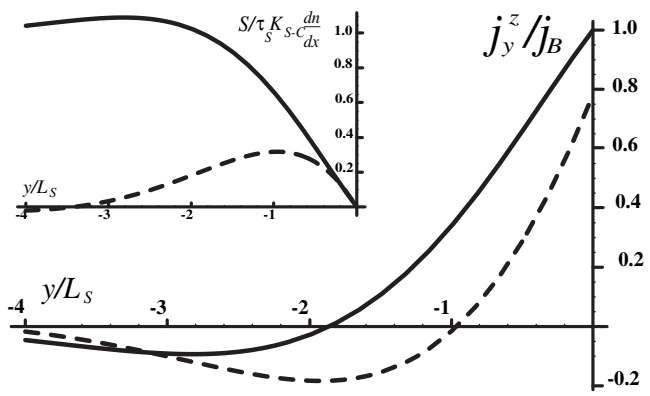

FIG. 2. SCs and accumulations in the R2DEG $(y<0)$ as a function of distance from the interface to the 2DEG. The full line is the local value of the SHC density for $\alpha k_{F} \tau / \hbar=0.1$. At the boundary the SHC density recovers its maximum value $j_{B}=$ $e E K_{s-c} / 2 \pi \alpha$ (it approaches the universal value $e E / 8 \pi$ in the clean limit $\xi \gg 1$ ). The dashed line is the $z$ component of the diffusion current density. Inset: Corresponding local SA in the same system normalized to the magnitude of the bulk SA $e E \alpha \tau m / 2 \pi$. The solid and dashed lines represent the $y$ and $z$ components, respectively. 
diffusion of the bulk $s_{y}$ SA. This is not a Hall current, since it flows into the side contacts with opposite directions (Fig. 2) with polarization that is inverted with the bias current direction. The resulting spin conductivity at the interface is $\sigma_{x y}^{y}=0.87 e \xi^{2} / 2 \pi$ in the dirty limit $(\xi \ll 1)$. For larger values of $\xi, \sigma_{x y}^{y}$ increases above this quadratic behavior, but in the clean limit $(\xi \gg 1)$ this increase is cut off by the resistance of the normal region. When spins diffuse from a finite distance into the 2DEG, they precess in the SO-generated magnetic field. Consequently, there is a diffusion current polarized along the $z$ direction, for which we find in the dirty limit a conductivity $\sigma_{x y}^{z}=$ $0.83 e \xi^{2} / 2 \pi$. The conductivity $\sigma_{x y}^{z}$, contrary to $\sigma_{x y}^{y}$, $d e-$ creases below this quadratic behavior for larger values of $\xi$ and vanishes in the clean limit. Nikolić et al. [21] recently observed SCs with $z$ and $y$ polarization in numerical simulations. In addition, we also find a SHC exponentially localized to the edges that decays in the bulk on the length scale $L_{s}$ and reaches its maximum value $e E K_{s-c} / 2 \pi \alpha$ at the interface to the reservoir. This is due to the fact that the first term in Eq. (9) being proportional to $s_{y}$ (thus zero at the interface) is no longer screening the second term (proportional to $\nabla n$ ) and reflects the physical process that the SHC density generated near the interface can escape into the reservoir before it decays due to spin relaxation. The resulting dc spin Hall conductivity is given by $e \xi^{2} / 2 \pi$ in the dirty and $e / 8 \pi$ in the clean limit. Our result differs from that of the authors of Ref. [13] who did not take into account the edge currents and obtained similar values only for the ac response at carefully tuned frequencies.

In conclusion, we find that in a Hall geometry two different spin currents can be extracted by the Hall contacts from the current-biased disordered R2DEG. In addition to the SHC, the current-induced SA drives a spin-diffusion current. The SO-generated spin accumulation is therefore not confined to the region where it is generated but can be extracted and, at least in principle, used as a source of spins for spintronics applications. Both diffusion and SHCs are generated within a strip that scales like the DyakonovPerel spin-diffusion length.

We thank Junichiro Inoue, Philip Stamp, Fei Zhou, and especially Yuli V. Nazarov for useful discussions. This work was supported by the FOM, EU Commission FP6 NMP-3 Project No. 05587-1 "SFINX," NSERC Canada discovery Grant No. R8000, and PITP.

*Present address: Department of Physics and Astronomy, University of British Columbia, 6224 Agricultural Road, Vancouver, BC, Canada V6T 1 Z1.

[1] I. Žutić et al., Rev. Mod. Phys. 76, 323 (2004).

[2] G. Schmidt et al., Phys. Rev. B 62, R4790 (2000).

[3] F. T. Vas'ko and N. A. Prima, Sov. Phys. Solid State 21, 994 (1979); L. S. Levitov et al., Zh. Eksp. Teor. Fiz. 88, 229 (1985) [Sov. Phys. JETP 61, 133 (1985)].
[4] V. M. Edelstein, Solid State Commun. 73, 233 (1990); J. I. Inoue et al., Phys. Rev. B 67, 033104 (2003).

[5] Y. K. Kato et al., Nature (London) 427, 50 (2004); Y. K. Kato et al., Phys. Rev. Lett. 93, 176601 (2004); in hole systems, A. Yu. Silov et al., Appl. Phys. Lett. 85, 5929 (2004); S. D. Ganichev et al., cond-mat/0403641.

[6] M. I. Dyakonov and V. I. Perel, JETP Lett. 13, 467 (1971).

[7] J. E. Hirsch, Phys. Rev. Lett. 83, 1834 (1999); S. Zhang, Phys. Rev. Lett. 85, 393 (2000); R. V. Shchelushkin and A. Brataas, Phys. Rev. B 71, 045123 (2005).

[8] S. Murakami et al., Science 301, 1348 (2003); Phys. Rev. B 69, 235206 (2004); for 2D hole systems: B. A. Bernevig and S.-C. Zhang, Phys. Rev. Lett. 95, 016801 (2005).

[9] J. Sinova et al., Phys. Rev. Lett. 92, 126603 (2004); J. Hu et al., Int. J. Mod. Phys. B 17, 5991 (2003); S.-Q. Shen, Phys. Rev. B 70, 081311(R) (2004); D. Culcer et al., Phys. Rev. Lett. 93, 046602 (2004); N. A. Sinitsyn et al., Phys. Rev. B 70, 081312 (2004); A. A. Burkov et al., Phys. Rev. B 70, 155308 (2004).

[10] Y. K. Kato et al., Science 306, 1910 (2004); J. Wunderlich et al., Phys. Rev. Lett. 94, 047204 (2005).

[11] J. I. Inoue et al., Phys. Rev. B 70, 041303 (2004).

[12] S. Murakami, Phys. Rev. B 69, 241202(R) (2004).

[13] E. G. Mishchenko et al., Phys. Rev. Lett. 93, 226602 (2004).

[14] E. I. Rashba, Phys. Rev. B 70, 201309(R) (2004).

[15] O. V. Dimitrova, Phys. Rev. B 71, 245327 (2005); O. Chalaev and D. Loss, Phys. Rev. B 71, 245318 (2005).

[16] A. Khaetskii, cond-mat/0408136; R. Raimondi and P. Schwab, Phys. Rev. B 71, 033311 (2005).

[17] K. Nomura et al., Phys. Rev. B 72, 165316 (2005).

[18] D. N. Sheng et al., Phys. Rev. B 72, 153307 (2005).

[19] J. Sinova, S. Murakami, S.-Q. Shen, and M.-S. Choi, condmat/0512054.

[20] E. I. Rashba, J. Supercond. 18, 137 (2005); S. Zhang and Z. Yang, Phys. Rev. Lett. 94, 066602 (2005).

[21] B. K. Nikolić et al., Phys. Rev. B 72, 075361 (2005); condmat/0506588.

[22] B. K. Nikolić et al., Phys. Rev. Lett. 95, 046601 (2005); O. Bleibaum, cond-mat/0503471 v2; G. Usaj and C. Balserio, cond-mat/0405065; Q. Wang et al., cond-mat/0505576; A. G. Mal'shukov et al., Phys. Rev. Lett. 95, 146601 (2005).

[23] See, e.g., T. Koga et al., Phys. Rev. Lett. 89, 046801 (2002).

[24] W. Kohn and J.M. Luttinger, Phys. Rev. 108, 590 (1957).

[25] Reference [15] shows that the $\mathrm{SH}$ current operator is related to the time derivative of the magnetization (rather than the acceleration). The SHE then vanishes for stationary SAs. We require only that the charge current be stationary. For instance, near boundaries the SA has been observed to precess at constant charge current [10].

[26] J. Schliemann et al., Phys. Rev. Lett. 90, 146801 (2003).

[27] F. T. Vas'ko, Zh. Eksp. Teor. Fiz. 79, 953 (1980) [Sov. Phys. JETP 52, 485 (1980)].

[28] Our matching conditions also hold for nonabrupt interfaces with varying $\alpha$, provided the thickness of the interface region is sufficiently smaller than the Dyakonov-Perel spin relaxation length. 